\title{
53 MHZ BEAM LOADING COMPENSATION FOR SLIP STACKING IN THE FERMILAB MAIN INJECTOR*
}

\author{
J. Dey ${ }^{\#}$, I. Kourbanis, FNAL, Batavia, IL 60510, U.S.A.
}

\section{Abstract}

Recently In-Phase and Quadrature (I\&Q) was added to both the $53 \mathrm{MHz}$ Feedback and Feedforward Beam Loading Compensation for Slip Stacking in the Fermilab Main Injector. With $53 \mathrm{MHz}$ Feedback, we can now turn the 18 Radio Frequency (RF) Stations off down to below $100 \mathrm{~V}$. In using I\&Q on Feedforward, beam loading compensation to the beam on both the upper and lower frequencies of Slip Stacking can be applied as we slip the beam. I\&Q theory will be discussed.

\section{IN-PHASE AND QUADRATURE THEORY}

The following is derived from the diagram of In-phase and Quadrature shown in Figure 1.

Letting A represent the phase of LO where $f_{\mathrm{LO}}$ is the down convert and up convert frequency and $t$ is time

$$
A=2 \pi f_{L O} t
$$

Next, $B$ represents the phase of $R F_{\text {in }}$ where $f_{R F}$ is the frequency coming into the system and $\theta(t)$ is an arbitrary phase offset of the incoming $R F_{\text {in }}$

$$
B=2 \pi f_{R F} t+\theta(t)
$$

One then can define $\mathrm{RF}_{\text {in }}$ to be

$$
R F_{i n}=\cos (B)
$$

The resulting down convert output is $\mathrm{LO} \times \mathrm{RF}_{\text {in }}$ In-phase

$$
\begin{aligned}
& \cos (A) \cos (B)= \\
& \frac{1}{2}(\cos (A-B)+\cos (A+B))
\end{aligned}
$$

Quadrature

$$
\begin{aligned}
& -\sin (A) \cos (B)= \\
& -\frac{1}{2}(\sin (A-B)+\sin (A+B))
\end{aligned}
$$

Sending the down convert output through a Low Pass Filter to remove the upper resulting frequency gives $\underline{\text { In-phase }}$

$$
\frac{1}{2} \cos (A-B)=\frac{1}{2} \cos (C)
$$

Quadrature

$$
-\frac{1}{2} \sin (A-B)=-\frac{1}{2} \sin (C)
$$

where $\mathrm{C}$ is defined as

$$
C=A-B
$$

Taking the Low Pass Filter output through the up convert results in

\section{$\underline{\text { In-phase }}$}

$$
\begin{aligned}
& \cos (A) \frac{1}{2} \cos (C)= \\
& \frac{1}{4}(\cos (A-C)+\cos (A+C))
\end{aligned}
$$

Quadrature

$$
\begin{aligned}
& -\sin (A) \frac{-1}{2} \sin (C)= \\
& \frac{1}{4}(\cos (A-C)-\cos (A+C))
\end{aligned}
$$

Summing up In-phase and Quadrature results in

$$
\begin{aligned}
& R F_{\text {out }}=\frac{1}{2} \cos (A-C)= \\
& \frac{1}{2} \cos (A-(A-B))
\end{aligned}
$$

Therefore

$$
R F_{\text {out }}=\frac{1}{2} \cos (B)
$$

Thus, the $\mathrm{RF}_{\text {out }}$ signal is the same as the $\mathrm{RF}_{\mathrm{in}}$ signal except for in magnitude.

\footnotetext{
*Operated by Universities Research Association, Inc. for the U.S Department of Energy under contract DE-AC02-76CH03000. "dey@fnal.gov
} 


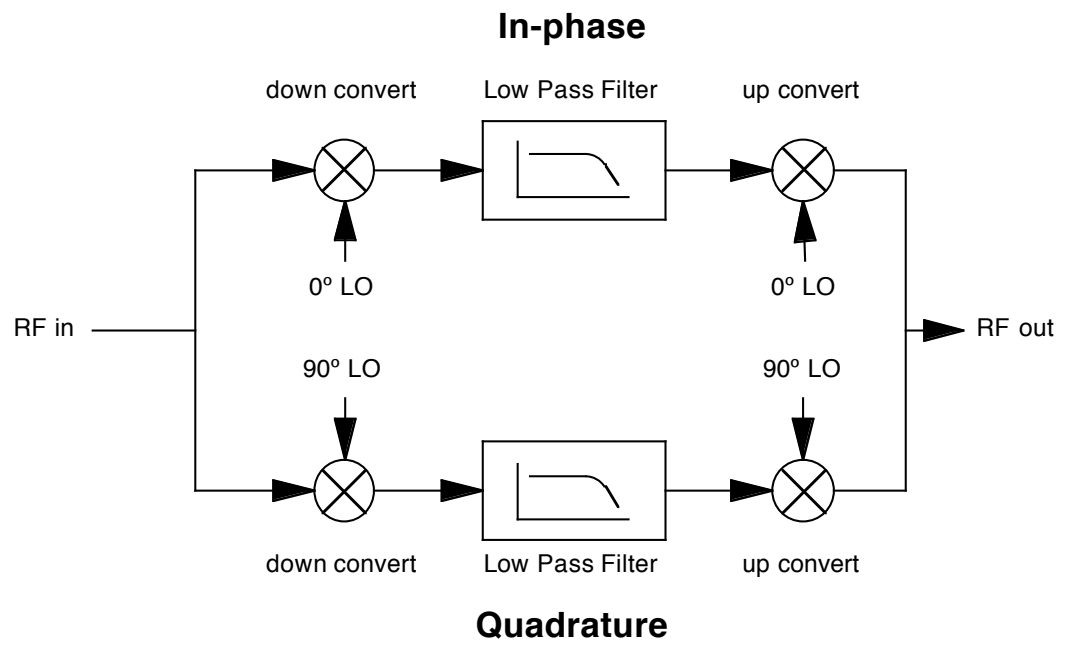

Figure 1: In-phase and Quadrature Diagram

The second result from this is that $\mathrm{RF}_{\text {out }}$ is independent of what $f_{\mathrm{Lo}}$ that you use. This is important because in the experimental applications of multiplication, a mixer leaks (typical isolation of a mixer is in the range of 50 to $60 \mathrm{~dB}$ ) through some of $f_{L O}$ even when being multiplied by zero. Now one can move the $f_{L O}$ to a frequency that can be filtered off after $\mathrm{RF}_{\text {out }}$ to preserve the integrity of $\mathrm{RF}_{\text {out }}$ to $\mathrm{RF}_{\text {in }}$.

The third important result is that one can put a Low Frequency Digital Time Delay between the Low Pass Filter and the up convert.

\section{SLIP STACKING}

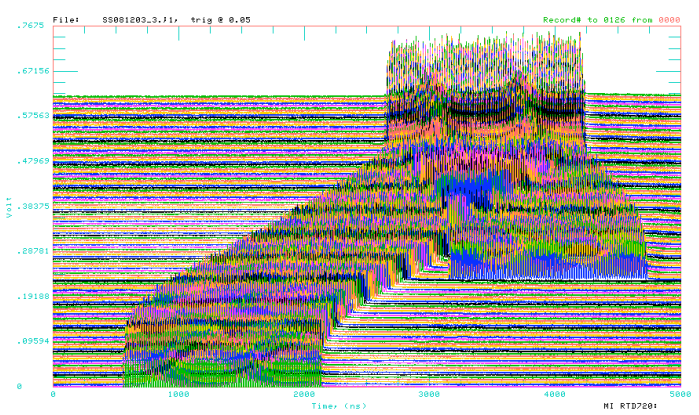

Figure 2: Mountain Range of Slip Stacking

It had been realized very early on that one could slip stack beam in the Main Injector (MI) at low intensities, but once any beam of any significant quantity (2e12 protons) was slipped, all of the signatures of beam loading on the 18 Main Injector $53 \mathrm{MHz}$ Cavities became apparent. The main criteria was to have the first nine cavities (Group A) and second nine cavities (Group B) each be able to supply $90 \mathrm{kV}$ of RF during slipping and to have each group operate at independent frequencies. The maximum separation in frequency between Group A and B is $1200 \mathrm{~Hz}$.

Presently we have MI Stations 8 and 9 in Group A and 17 and 18 in Group B produce the $90 \mathrm{kV}$ required for their respective group during slipping. The other 14 stations are turned off during this. In order to do slip stacking at high intensities [1] (Figure 2), I\&Q was applied to the direct RF feedback of the station for stations off and to the feedforward beam loading compensation on all of the stations because of each groups individual slipping frequency.

\section{Feedback}

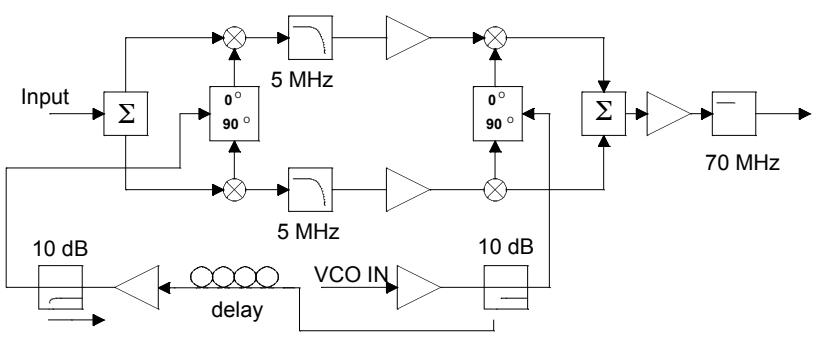

Figure 3: Beam Loading Compensation Filter Module

Direct RF feedback is applied at each individual station. The main problem we were having was in the up convert on Figure 3 [2] because the VCO IN was the same low level RF (LLRF) signal that we use to drive the cavities. The isolation of VCO IN to the mixer is only in the $50 \mathrm{~dB}$ range and was bleeding through enough to produce almost $1 \mathrm{kV}$ at the gap when the station LLRF was gated off.

In order to reduce the bleed through on a given station a new VCO IN was created for Figure 3 and is shown below in Figure 4 as RF out.

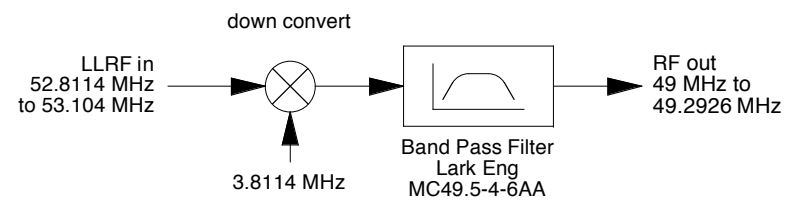

Figure 4: Down Convert of LLRF

The Lark Engineering MC49.5-4-6AA band pass filter effectively cuts off any frequency above $56 \mathrm{MHz}$ by 60 $\mathrm{dB}$ while passing the new VCO IN, $49 \mathrm{MHz}$ to 49.2926 
$\mathrm{MHz}$ signal. Only a slight change (less than $18 \mathrm{~ns}$ ) in the delay line was added to close the direct RF feedback loop around the station. The direct RF feedback loop then was checked at $52.8114 \mathrm{MHz}$ and $53.104 \mathrm{MHz}$ to ensure proper operation. This effectively works because the new VCO IN of $49 \mathrm{MHz}$ is still bleeding through but with the $53 \mathrm{MHz}$ cavities having a Q of $2500(8 \mathrm{GeV})$ to 5000 $(150 \mathrm{Gev})$ it is filtered off. The last upgrade for stations off was to replace the original mixer ( $56 \mathrm{~dB}$ of isolation) that was used to shut the LLRF off to the station with a Mini-Circuits ZASWA-2-50DR GaAs SPDT switch with a measured $85 \mathrm{~dB}$ of isolation.

Below in Figure 5 is a plot done on a HP 89441A Vector Signal Analyzer (VSA) of the fan-back sum of all $18 \mathrm{MI}$ Stations at $52.8114 \mathrm{MHz}(8 \mathrm{GeV})$ with stations off and no beam in the MI. The result is $-77.643 \mathrm{dBm}$ and equates to $88.58 \mathrm{~V}$ when one knows that the attenuation factor is $126.59 \mathrm{~dB}$ from the gap monitor to the summation. This equates to $4.9 \mathrm{~V}$ per station.

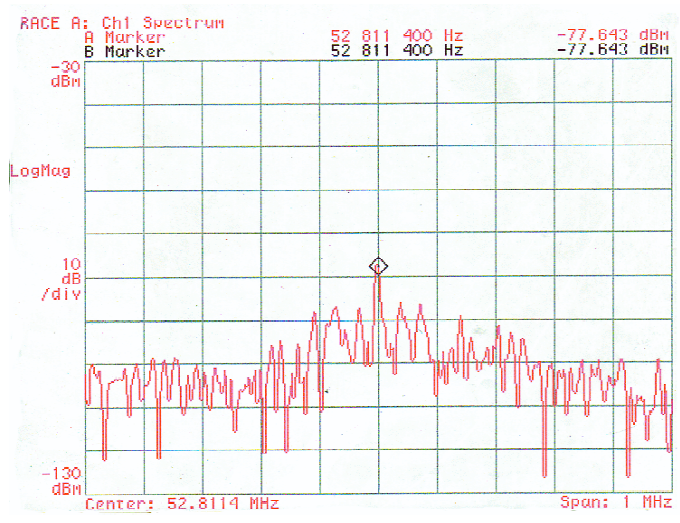

Figure 5: Stations Off Fan-back Sum of all 18 MI Cavities

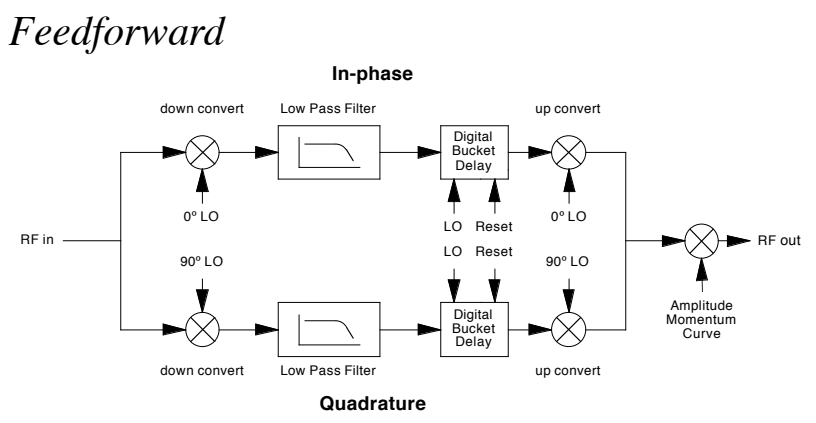

Figure 6: Feedforward Beam Loading Compensation

Figure 6 is the block diagram of the new Feedforward Beam Loading Compensation [3] that is fanned out to each of the 18 stations. RF out from Figure 4 is now LO for Figure 6. The Digital Bucket Delay is set to 494 and is in terms of the LO rf cycles. In using LO, the Feedforward now completely tracks the beam as it goes through the frequency sweep up the ramp from $8 \mathrm{GeV}$ to $150 \mathrm{GeV}$. The Low Pass Filter in Figure 6 is a MiniCircuits BLP-30 (30 MHz) and an Amplitude Momentum
Curve is used to electronically attenuate the signal for different gains and number of bunches.

The new Feedforward Beam Loading Compensation accomplishes three things. First, it allows us to apply the beam loading compensation during the slip stacking frequency separation. Second, the beam loading compensation tracks the beam up the ramp and third, the $49 \mathrm{MHz}$ bleed through on the up convert does not affect stations off because it is filtered off by the cavity. This coupled with increasing the cathode drive to the Y-567B from $4 \mathrm{~kW}$ to $8 \mathrm{~kW}$ and programming the grid of the Y567B from $-275 \mathrm{~V}$ to $-175 \mathrm{~V}$ during slipping gives us the beam loading compensation shown in Figure 7 for Slip Stacking.

Figure 7 shows the VSA plot of the gap monitor on Main Injector Station number two during the slipping process. It is one of the seven stations in Group A that are off during the slipping process and the beam intensity level is at $7.5 \mathrm{e} 12$ protons. The blue trace is the beam loading induced on the station from the beam and the green trace is when feedforward beam loading compensation is applied. Note that $23.4 \mathrm{~dB}$ of compensation is obtained and there is no bleed through from the up convert on the fundamental frequency from either feedback or feedforward.

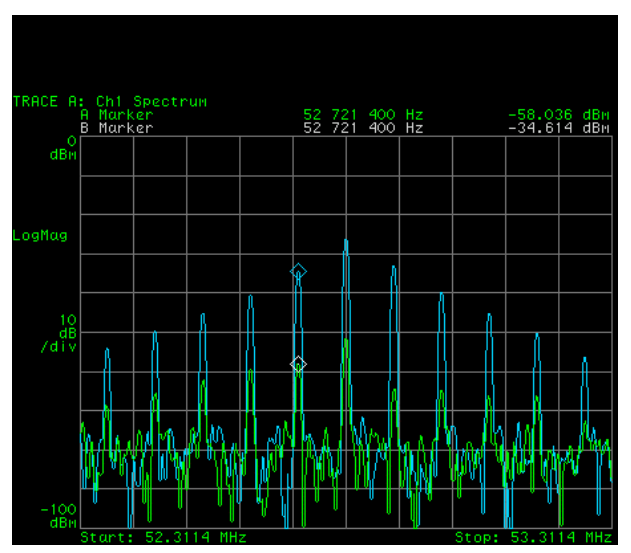

Figure 7: Gap Monitor on MI Station \#2 during Stations Off while Slip Stacking

\section{ACKNOWLEDGEMENT}

Special thanks to John Reid and Tim Berenc for their work on making the $4 \mathrm{~kW}$ to $8 \mathrm{~kW}$ Solid State Driver upgrade a reality.

\section{REFERENCES}

[1] K. Seiya, et al, "Status of Slip Stacking at Fermilab Main Injector," 2005 PAC, these proceedings

[2] J. Dey, J. Steimel, J. Reid, "Narrowband Beam Loading Compensation in the Fermilab Main Injector Accelerating Cavities," 2001 PAC, p. 876, Chicago, June 2001

[3] J. Dey, et al, "53 MHz Feedforward Beam Loading Compensation in the Fermilab Main Injector," 2003 PAC, p. 3353, Portland, May 2003 